\title{
A Review on Classification and Comparison of Automatic Logo Based Document Image Retrieval Methods and other Applications
}

\author{
Raveendra.K \\ Research Scholar, Department of Electronics and Communication Engineering, \\ Koneru Lakshmaiah University, Green fields, Vaddeswaram, Guntur District, \\ Andhra Pradesh, India, EmailId:raveendratech@gmail.com, \\ Orcid Id: 0000-0001-9695-1908 \\ Dr. P V N Reddy \\ Professor and Principal, Department of Electronics and Communication Engineering, \\ Sri Venkateswara College of Engineering, Balaji Nagar, Kadapa, Kadapa District, \\ Andhra Pradesh, India. \\ EmailId:principal@svck.edu.in \\ Dr. P V V Kishore \\ Professor, Department of Electronics and Communication Engineering, \\ Koneru Lakshmaiah University, Green fields, Vaddeswaram, Guntur District, \\ Andhra Pradesh, India. \\ Email Id:pvvkishore@gmail.com
}

\begin{abstract}
In this paper we provided an effective categorization of vast number of methods, techniques, transforms, algorithms, approaches and schemes available for the purpose of logo detection in various image processing applications like Authenticity of documents, Security for information, Traffic Surveillance (Intelligent Transportation System), Pattern recognition, Marketing, Medical Imaging, Satellite imaging, Drones and Ocean Imaging. We have highlighted the mostly used Scale Invariant Feature Transform (SIFT) and Speed Up Robust Feature (SURF) Algorithms along with the comparison between these two (SIFT and SURF) algorithms.
\end{abstract}

Keywords: ART,AMI, RANSAC, RDHOG, RTMI, LPT FMT, GLOH.

\section{INTRODUCTION}

The logo is an Authentication, Trade mark, Discriminator and Uniqueness for the Institutions, Companies, Organizations and some agency respectively. Just by observing the logo in a document it can be decided that is belongs to a particular Institution or Company or Organization or some Agency. For example if we have an Apple image on any electronic gadget or document immediately we can say that gadget or document belongs to the Apple Company. In the present rapidly increasing data environment through Internet, The necessity for this data maintenance, accessing, security and transparency should be continuously increased. For this purpose the image processing techniques like Automatic logo or signature or image (which provides the identity, authenticity, trademark, easy discrimination and consists of large amount of information in minimum space-one image consists of 10000 words) based document retrieval methods plays a vital role. Using these automatic logo based document image retrieval methods the data management will become easy specifically in the indexing of website contents and their maintenance. Till now, very vast number of methods, techniques, transforms and algorithms were proposed for the automatic Logo based Document Image Retrieval and other applications.

In this paper, we covered almost all the methods, classified them into different categories based on their applications. And finally we have done the comparison of the mostly used and advanced methods with their basic versions. Remaining flow order of this paper was organized into as follows. In the next section global classification of logos was covered. Section III gives classification of all the methods, techniques, transforms and algorithms based on various applications. Section IV provides the comparison between most popularly used and advanced techniques. Section V Summarizes the related work done so far. The conclusion was given section VI and last section VII ends with acknowledgement.

\section{GLOBAL CLASSIFICATION OF LOGOS}

A logo is a two dimensional complex image with different shapes and may consist of textual, symbolic, graphical, colour and combination of all with some other features with various characteristic appearances also. Therefore the identification and extraction of this logos in documents is a very challenging task in almost all types of digital image applications. The images can be classified globally into three categories as Logos with only text, Logos with only symbols and Logos with text and Symbols based on the very fundamental characteristics: Text and symbols. More over the images can also be classified 
based on based the other features like shape, colour and graphical properties also as shown in figure1.

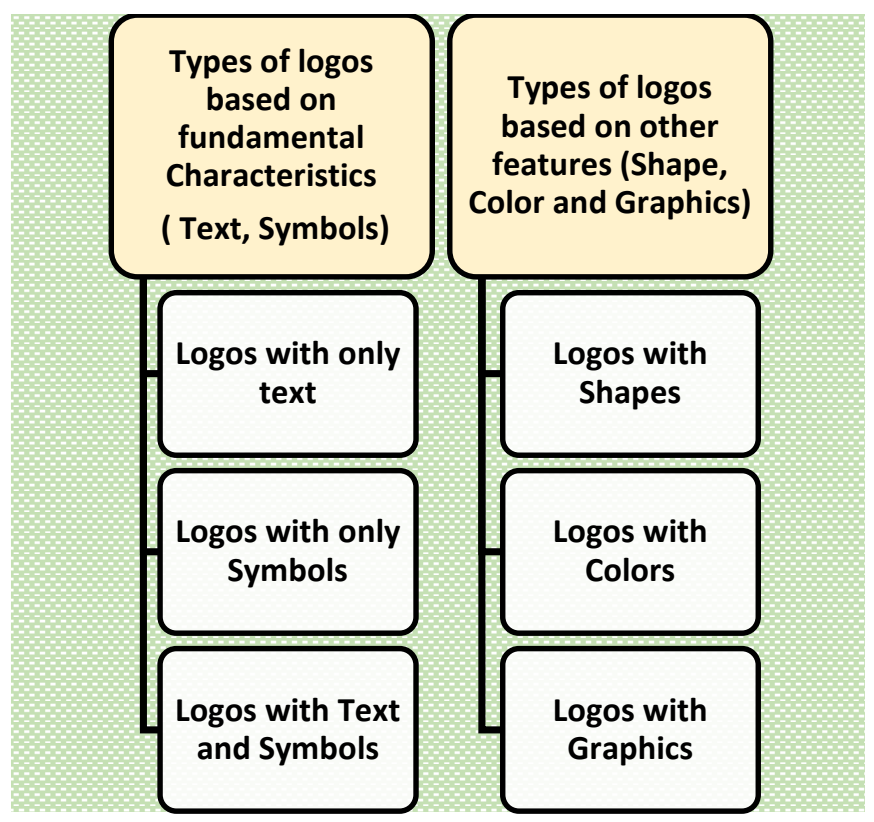

Figure 1: Classification of Logos

\section{Classification of all Methods}

The various methods, techniques, transforms, algorithms, Approaches and schemes covered in almost all the previous papers can be broadly classified into Three groups (i) For Document Retrieval Purpose, (ii) For Intelligent Transportation Systems and (iii) For Motional Video applications as shown in the figure 2 .

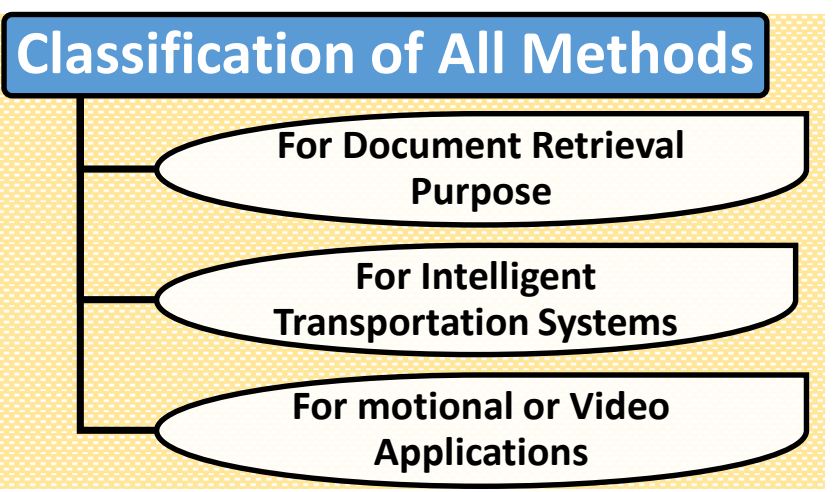

Figure 2: Classification of all Methods

(i). For Document Retrieval Purpose:

- Segmentation techniques

- Wavelets(DWT,DCT,FFT)

- $\quad$ Scale Invariant Feature Transform(SIFT)

- $\quad$ Speed Up Robust Feature(SURF) Algorithm

- Binary Robust Independent Elementary Features(BRIEF)
- $\quad$ Oriented FAST and Rotated BRIEF(ORB)

- Local Binary Patterns(LBR)

- Synthetic Context Logo (SCL) Image generation.

- $\quad$ State Vector Machine (SVM)

- $\quad$ K-Nearest Neighbours(KNN)

- Histogram Oriented Technique(HoG)

- Context Dependent Similarity(CDS)

- RANdom SAmple Consensus(RANSAC)

(ii) For Intelligent Transportation Systems (ITS):

- $\quad$ Logistic Regression(LR)

- $\quad$ Histogram of Oriented Gradients(HOG)

- Pyramid of Histograms of Orientation Gradients (PHOG)

- Relative Discriminative extension to HoG (RDHOG)

- $\quad$ SIFT\&SURF Algorithms

- Regions with Convolutional Neural Networks(RCNN)

- $\quad$ Support Vector Machine (SVM)

- $\quad$ Latent Support Vector Machine(LSVM)

- $\quad$ Conjugate Gradient Descent(CGD)

- Multi-scale Block Local Ternary Patterns(MB-LTP)

- Iterated Conditional Mode(ICM) Algorithm

- Markov Random Field(MRF) frame work

- Gaussian Mixed Model(GMM)

- Kernel Density Estimation(KDE)

- Principal Component Analysis-SIFT(PCA-SIFT)

(iii) For Motional and Video Applications:

- $\quad$ SIFT\&SURF Algorithms

- $\quad$ Support Vector Machine (SVM)

- Method of Moments

- Angular Radial Transform(ART)

- Affine Moment Invariant(AMI)

- Zernike Moments(ZM)

- Tchebichef Moments (TM)

- $\quad$ Radial Tchebichef Moments (RTM)

- $\quad$ Radial Tchebichef Moment Invariants (RTMI)

- Krawtchouk Moment(KM)

- Krawtchouk Moment Invariants(KMI) 
International Journal of Applied Engineering Research ISSN 0973-4562 Volume 12, Number 24 (2017) pp. 15458-15463

(C) Research India Publications. https://dx.doi.org/10.37622/IJAER/12.24.2017.15458-15463

- $\quad$ Legendre Moment(LM)

- $\quad$ Zernike Moment(ZM)

- $\quad$ Pseudo-Zernike Moment(PZM)

- Voting based Decision Scheme

- Hough Transform

- Color Edge Co-occurrence Histogram(CECH)

- Spatial Connected Component Descriptor(SCCD)

- Auto associator based Artificial Neural Networks(AANN)

- $\quad$ Outer Contour Strings(OCS)

- Log-Polar Transform(LPT)

- $\quad$ Fourier Mellin Transform(FMT)

- Gradient Location-Oriented Histogram(GLOH)

\section{Comparison of Advanced Methods}

Even though there are vast number of methods, techniques, transforms, algorithms, approaches and schemes covered in the previous papers, the mostly used are SIFT, SURF and Moment Invariant methods only with some enhanced characteristics for almost all types of applications due to their high performance nature. The Scale Invariant Feature Algorithm (SIFT) was first introduced by David G.Lowe in the year 2004 and became very popular because of it high performance index with respect to scale, rotation, intensity and illumination properties of the logo or image of both static and dynamic (moving) forms. The SIFT consists of four major steps as (i) Scale Space Peak or Extreme Points Detection, (ii) Localization of Key Points, (iii) Orientation Assignment and (iv) Generation of Descriptor as shown in the figure 3.

\section{Scale Space Peak or Extreme points selection}

\section{Localization of Key Points}

Orientation assignment

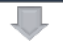

Generation of Descriptor

Figure 3: SIFT Algorithm

The SUFT consists of six major steps as (i) Fast Hessian Detection, (ii) Construction of Scale Space, (iii) Localization of Accurate Interested Points, (iv) Interested Point Descriptor, (v) Orientation Assignment and (vi) Descriptor Components as shown in the figure 4.

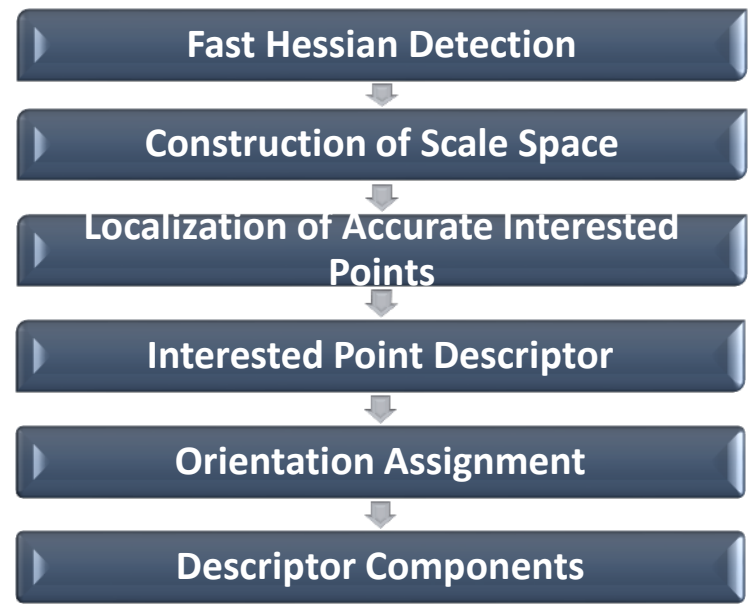

Figure 4: SURF Algorithm

SURF is enhanced version of SIFT and performs faster than SIFT without any reduction in the quality of the interested points. Binary Robust Independent Elementary Features (BRIEF) is an alternative of SIFT with somewhat less complexity. Oriented fast and Rotated BRIEF (ORB) is also another alternative for SIFT and SURF. In all these methods the difference is how they will identify the key points in the logo or image which are invariant to scale, rotation, intensity and illuminations using different techniques like K-Nearest Neighbours (KNN), State Vector Machine (SVM), Wavelet Transforms, Filters and algorithms depending on the particular application. For applications like Intelligent Transportation Systems (Vehicles Identification, Number plates recognition, Traffic Surveillance, Road Surveillance etc.), Television Videos, Sport Videos, Animations, Drones and other movement related areas or applications the above methods can be used in association different moment Invariant properties detection techniques which include Radial Tchebichef Moment Invariants (RTMI), Radial Tchebichef Moments (RTM), Tchebichef Moment (TM), Krawtchouk moments (KM), Krawtchouk moment Invariants (KMI), Legendre Moment (LM), Pseudo-Zernike Moments(PZM), Zernike Moments (ZM) and Affine Moment Invariants (AMI).

\section{RELATED WORK}

In [1] Ruilong Chen, Olga Isupova and Hao Zhu applied Cauchy Prior Logistic Regression (CPLR) method combined with Conjugate Gradient Descent (CGD) for the Online Vehicle Logo Recognition and got an accuracy of $98.8 \%$ for both small and large data sets.

In [2] Kazunori Aoki and Tsu-Shi Proposed the method Maximally Stable External Regions (MSER) along with 
gradient histogram and System Vector Machine (SVM) for automatic extraction and recognition of shoe logos effectively.

In [3] Afsoon Asghari Shirazi, Alireza Dehghani, Hasan Farsi and Mehran Yazdi used Local Binary Pattern features for the recognition of Persian Logos with their experimental result accuracy of $98 \%$.

In [4] Hang Su, Xiatian Zhu and Shaogang Gong used Synthetic Context Logo (SCL) image generation method for deep learning of logo detection models.

In [5] Ma'moun Al-Smadi, Khairi Abdulrahim and Rosalina Abdul Salam provided a detailed review on computer vision techniques used for Traffic Surveillance and monitoring systems.

In [6] Yizhang Xia, Jing Feng and Bailing Zhang used Multitask Learning with CNN (ML-CNN) for vehicle logo recognition and prediction of attributes and achieved overall accuracy of $98.14 \%$.

In [7] B.V. Dhandra, Sridevi Soma and Gururaj Mukarambi worked on Identification of Institutional logos using DWT and FFT features with SVM Classifiers to identify the logos in large number of data sets.

In [8] Ms.Sushma H.Bailmare and Prof.A.B.Gadicha done a review on vehicle number plate recognition using Segmentation method.

In [9] N.Vinay Kumar, Pratheek, V.Vijay Kantha, K.N. Govindaraju and D.S.Guru used KNN Classifier for the classification of logos into pre-defined classes.

In [10] Sina Hassanzadeh and Hossein Pourghassem proposed a novel fast method using morphological features and decision tree classifier for logo detection.

In [11] Cong Chen, Xiaobo Lu, Shengqin Jiang and Jiaji Song used two feature extraction methods Pyramid of Histograms of Orientation Gradients (PHOG) and Multi-scale Block Local Ternary Patterns (MB-LTP) for the effective recognition of road surveillance images.

SIFT and SURF algorithms were used in [12] to [20].

In [12] Sheena $S$ and Sheena Mathew compared SIFT and SURF algorithms for the iris recognition along with KNN algorithm for classification.

In [13] Qin Gu, Jianyu Yang, Guolong Cui, Lingjiang Kong, Huakun Zheng and Reinhard Klette used a directional SIFT flow parsing method for multi scale vehicle logo recognition and obtained the evaluated performance of about $97.4 \%$.

In [14] He Jingmeng, Xie Yuxiang, Luan Xiado, Niu Xiao and Zhang Xin used SURF and Bag-of-Words method for TV Logo detection and recognition with the support of KNN and SVMs.

In [15] Mrs. Poonam Kondekar and Prof. Priti Shende recognized the logos using Context dependent criteria by using Context Dependent Similarity (CDS-SIFT) and CDS-
RANSAC (RANdom Sample Consensus) algorithms and finally stated that the accuracy of RANSAC method is more than CDS-SIFT.

In [16] Ebrahim Karami, Siva Prasad and Mohamed Shehata compared the performances of SIFT, SURF, BRIEF and ORB methods for distorted images based on the parameters: number of key points, matching rate and execution time required.

In [17] Chinmoy Biswas and Joydeep Mukherjee used SIFT, SURF and HOG descriptors for the logo recognition.

In [18] P M Panchal, S R Panchal and S K Shah compared SIFT and SURF algorithms

In [19] Nagham Hamid, Abid Yahya, R.Badlisha Ahmad and Osamah M. Al-Qershi used and compared SIFT and SURFs for characteristic region based image steganography with 1-level DWT and 2-level DWTs for Lena, Bridge and Pepper images.

In [20] Prof. Mrunalinee Patole and Meera Sambhaji Sawalkar used CDS-SIFT method in their improved approach for logo detection and recognition.

In [21] Zili Zhang, Xuan Wang, Waqas Anwar and Zoe L.Jiang compared the performances of almost all types of moments for the logo recognition.

In [22] Wei Zhang Q.M.J Wu,Guanghui Wang and Xinge You proposed a night time traffic surveillance system for vehicle headlight detection, tracking and pairing using Laplacian of Gaussian (LoG) filter, Markov Random Field (MRF) frame work and Iterational Conditional Mode (ICM)Algorithm.

In [23] Omar Mahammed Wahdan, Khairuddin Omar and Mohammad Faidzul Nasrudin developed a system for logo recognition using Angular Radial Transform (ART) along with Eucledian Distance as a similarity measuring parameter.

In [24] Serge Belongie, Jitender Malik and Jan Puzicha presented a novel approach for measuring similarity between shapes for object recognition.

In [25] Souvik Ghosh and Ranjan Perekh proposed an automated system for rotation and scale invariant color recognition using first and second order Invariant moments and also with four color moments: mean, standard deviation, skewness and kurtosis for the recognition of color logo images.

\section{CONCLUSION}

In this paper we reviewed almost all types of methods, techniques, transforms, algorithms, approaches and schemes with an effective classification and comparison between the mostly used two methods: SIFT and SURF in more number of Image Processing applications. And we hope that this detailed review on all methods will definitely create a road map for further research in latest advancements like Animation, Web Indexing and Drones. 


\section{ACKNOWLEDGEMENT}

I am highly indebted to my guides Dr. P V N Reddy and Dr. P $\mathrm{V} \mathrm{V}$ Kishore for their support and encouragement in the completion of this effective Literature review on almost all methods available for Image processing applications.

\section{REFERENCES}

[1] Ruilong Chen, Olga Isupova and Hao Zhu, "Online Vehicle Logo Recognition using Cauchy Prior Logistic Regression," $20^{\text {th }}$ International Conference on Information Fusion, Xi'an, China-July 10-13, 2017.

[2] Kazunori Aoki and Tsu-Shi, "Automatic Extraction Recognition of Shoe Logos with a Wide Variety of Appearance," 2017 Fifteen IAPR International Conference on Machine Vision Applications (MVA), Nagoya University, Nagoya, Japan, May 8-12,2017.

[3] Afsoon Asghari Shirazi, Alireza Dehghani, Hasan Farsi and Mehran Yazdi, "Persian Logo Recognition using Local Binary Patterns," $3^{\text {rd }}$ International Conference on Pattern Recognition and Image Analysis (IPRIA 2017) April 19-20, 2017.

[4] Hang Su, Xiatian Zhu and Shaogang Gong, "Deep Learning Logo Detection with Data Expansion by Synthesising Context," arXiv:1612.09322v2 [cs.CV] 4 Jan 2017.

[5] Ma'moun Al-Smadi, Khairi Abdulrahim and Rosalina Abdul Salam, "Traffi Vehicle Detection, Recognition and Tracking," International Journal of Applied Engineering Research, Volume 11, Number (1) (2016) pp 713-726.

[6] Yizhang Xia, Jing Feng and Bailing Zhang, "Vehicle Logo Recognition and Attributes Prediction by Multitask Learning with CNN," $201612^{\text {th }}$ International Conference on Natural Computation, Fuzzy Systems and Knowledge Discovery, IEEE, 2016.

[7] B.V. Dhandra, Sridevi Soma and Gururaj Mukarambi, "Identification of Institutional Logo based on Wavelet Features," International Journal of Computer Applications, Volume 107, No 15, December 2014.

[8] Ms.Sushma H.Bailmare and Prof.A.B.Gadicha, “ A Review paper on Vehicle Number Plate Recognition (VNPR) Using Improved Character Segmentation Method," International Journal of Scientific and Research Publications, Volume 3, Issue 12, December 2013.

[9] N.Vinay Kumar, Pratheek, V.Vijay Kantha, K.N. Govindaraju and D.S.Guru, "Features Fusion for Classification of Logos," International Conference on Computational Modeling and Security, Elsevier B.V., Cross Mark, doi:10.1016/j.procs.2016.
[10] Sina Hassanzadeh and Hossein Pourghassem, "Fast Logo Detection Based on Morphological Features in Document Images," IEEE $7^{\text {th }}$ International Colloquium on Signal Processing and its Applications, 2011.

[11] Cong Chen, Xiaobo Lu, Shengqin Jiang and Jiaji Song, "An Effective Vehicle Logo Recognition Method for Road Surveillance Images," $2^{\text {nd }}$ IEEE International Conference on Computer and Communications, 2016.

[12] Sheena S and Sheena Mathew, "A Comparison of SIFT and SURF Algorithm for the Recognition of an Efficient Iris Biometric System," International Journal of Advanced Research in Computer and Communication Engineering, Vol 5, Special Issue 1, February 2016.

[13] Qin Gu, Jianyu Yang, Guolong Cui, Lingjiang Kong, Huakun Zheng and Reinhard Klette, " Multi Scale Vehicle Logo Recognition By Directional Dense SIFT Flow Parsing," ICIP, IEEE, 2016.

[14] He Jingmeng, Xie Yuxiang, Luan Xiado, Niu Xiao and Zhang Xin, "A TV Logo Detection and Recognition Method Based on SURF Feature and Bag-of-Words Model," $2^{\text {nd }}$ IEEE International Conference on Computer and Communications, 2016.

[15] Mrs. Poonam Kondekar and Prof. Priti Shende, "Logo Recognition using Context Dependent Criteria," International Conference on Advances in Electronics, Communication and Computer Technology (ICAECCT), IEEE, 2016.

[16] Ebrahim Karami, Siva Prasad and Mohamed Shehata, "Image Matching using SIFT, SURF, BRIEF and ORB: Performance Comparison for Distorted Images," Newfoundland Electrical and Computer Engineering Conference at St.John's , Canada, November, 2015.

[17] Chinmoy Biswas and Joydeep Mukherjee, "Logo Recognition Technique using Sift Descriptor, Surf Descriptor and Hog Descriptor," International Journal of Computer Applications, Volume 117, N0.22, May 2015.

[18] P M Panchal, S R Panchal and S K Shah, "A Comparison of SIFT and SURF," International Journal of Innovative Research in Computer and Communication Engineering, Vol 1, Issue 2, April 2013.

[19] Nagham Hamid, Abid Yahya, R.Badlisha Ahmad and Osamah M. Al-Qershi, "A comparison between Using SIFT and SURF for Characteristic Region Based Image Steganography," International Journal of Computer Science Issues, Vol. 9, Issue 3, No 3, May 2012.

[20] Prof. Mrunalinee Patole and Meera Sambhaji Sawalkar, "Improved Approach for Logo Detection and Recognition," International Journal of Emerging Trends 
International Journal of Applied Engineering Research ISSN 0973-4562 Volume 12, Number 24 (2017) pp. 15458-15463

(C) Research India Publications. https://dx.doi.org/10.37622/IJAER/12.24.2017.15458-15463

\& Technology in Computer Science, Volume 3, Issue 6, November-December 2014.

[21] Zili Zhang, Xuan Wang, Waqas Anwar and Zoe L.Jiang, "A Comparison of Moments-Based Logo Recognition Methods," Hindawi Publishing Corporation, Abstract and Applied Analysis, Volume 2014. http://dx.doi.org/10.1155/2014/854516

[22] Wei Zhang Q.M.J Wu,Guanghui Wang and Xinge You, "Tracking and Pairing Vehicle Headlight in Night Scenes," IEEE Transactions on Intelligent Transportation Systems, Vol. 13, No. 1, March 2012.

[23] Omar Mahammed Wahdan, Khairuddin Omar and Mohammad Faidzul Nasrudin, "Logo Recognition System Using Angular Radial Transform Descriptors," Journal of Computer Science 7 (9), Science Publications, 2011.

[24] Serge Belongie, Jitender Malik and Jan Puzicha, "Shape Matching and Object Recognition Using Shape Contexts," IEEE Transactions on Pattern Analysis and Machine Intelligence, Vol. 24, No. 24, April 2002.

[25] Souvik Ghosh and Ranjan Perekh, "Automated Color Logo Recognition System based on Shape and Color Features," International Journal of Computer Applications, Volume. 118, No. 12, May 2015. 